\title{
Why change?
}

\author{
John W. Hammon, MD
}

This provocative editorial seeks to convince the readership that hyperkalemic depolarizing cardiac arrest afforded by potassium-containing cardioplegic solutions can be deleterious to cardiac function and may be responsible for some of the untoward effects of ischemic arrest often used for cardiac surgical operations today. Dr Dobson provides evidence that depolarizing cardiac arrest can have several deleterious effects, including alterations in the sodium/calcium homeostasis, vasoconstriction, and direct cellular injury provoking an inflammatory response. He further suggests that a compound composed of 2 molecules, specifically adenosine and lidocaine, can achieve the same electromechanical arrest at higher concentration than that used for reducing inflammation and other effects while avoiding some of the deleterious effects of hyperkalemia.

The potential problems associated with potassium cardioplegia have been discussed for some time, and 2 particularly good articles are referenced by Dr Dobson (references 5 and 17).

Perhaps the most important feature of the cardioplegia controversy is that there is no great impetus for change in this country or the world today because the results of cardiac surgery have improved to the point where it would take a profound improvement to alter morbidity and mortality outcomes. Many surgeons, including our group, use an arresting concentration of potassium $(25 \mathrm{mEq} / \mathrm{L})$ in a buffered blood solution and switch to a lower concentration of potassium $(10 \mathrm{mEq} / \mathrm{L})$ for subsequent doses delivered intermittently antegrade and retrograde. High volumes of potassium derived from cardioplegia can cause systemic hyperkalemia, which can be deleterious in patients with renal insufficiency. Likewise, the emergence of cardiac electrical activity associated with too little cardioplegia can cause ischemic myocardiac damage.

In the real world of cardiac surgery, many operations with ischemic arrest periods of more than 90 minutes can be carried out in patients with normal ventricles with little, if any, evidence of cardiac damage postoperatively. Therefore, why the interest in a new compound? Almost all car-

\footnotetext{
From the Department of Cardiothoracic Surgery, Wake Forest University School of Medicine, Winston-Salem, NC.

Received for publication June 29, 2010; accepted for publication July 3, 2010.

Address for reprints: John W. Hammon, MD, Wake Forest University School of Medicine, Department of Cardiothoracic Surgery, Medical Center Boulevard, Winston-Salem, NC 27157-1096 (E-mail: jhammon@wfubmc.edu). J Thorac Cardiovasc Surg 2010;140:1218-9

$0022-5223 / \$ 36.00$

Copyright (c) 2010 by The American Association for Thoracic Surgery doi:10.1016/j.jtcvs.2010.07.012
}

diac surgeons have had the experience of operating on patients with poor left ventricular function that required extensive cardiac surgery in which significant myocardial dysfunction occurred postoperatively. This is particularly true if some of the preoperative myocardial injury is acute. In these cases, high doses of inotropic drugs, aortic balloon pump assist, or left ventricular assist device implantation may be necessary to preserve life. This does not happen often, but when it does it often ends with a disappointing result. Is this because of the toxic effects of cardioplegia, ischemic arrest, or both? The answer is not exactly known, but any surgeon who has been in that situation would wish for better myocardial protection.

A recent study of perioperative ischemia demonstrated low but significant elevations in cardiac isoenzymes associated with ischemic arrest and hyperkalemic cardioplegia. ${ }^{1}$ This would make one suspicious that a low level of myocardial damage is produced during hyperkalemic cardioplegic protection from ischemic arrest that may not become manifest unless significant myocardial dysfunction was present preoperatively.

So what needs to be done in 2010 ? There are many anecdotal statements from surgeons who have used the combination of adenosine and lidocaine now marketed under the trademark of Adenocaine (Hibernation Therapeutics, Wicklow, Ireland). Because there has been no significant clinical research for this compound, I would vote for 2 distinct phases of investigation. In the first, this drug could be used in crystalloid cardioplegia or with blood (4:1 blood:crystalloid or microplegia formulations) and varying combinations of potassium and other additives to investigate the animal model of acute myocardial ischemia and reperfusion, as suggested and used by Dr Jakob VintenJohansen to develop better myocardial protection techniques for the sickest hearts. ${ }^{2}$

In addition, one of the significant needs in our profession is for the ability to preserve the heart destined for transplantation. Significant preliminary evidence is presented in this editorial for a role of Adenocaine in improved preservation of the donor heart. I would therefore suggest that an experimental protocol be used to test this nondepolarizing arrest on a transplantation animal model. If these animal studies are promising, then we could rapidly move into a clinical testing phase in both the arenas of damaged hearts for repair and preservation of the heart for transplantation.

We should be grateful that Dr Dobson and associates have persisted in their work and laid the groundwork for perhaps 
a significant improvement in our ability to surgically treat patients with severe heart disease.

\section{References}

1. Mangano DT, Maio Y, Tudor IC, Investigators of the Multicenter Study of Perioperative Ischemia (McSPI) Research Group, Ischemia Research and Education
Foundation (IREF). Post-reperfusion myocardial infarction: long-term survival improvement using adenosine regulation with acadesine. J Am Coll Cardiol. 2006;48: 206-14.

2. Muraki S, Morris C, Budde JM, Zhao ZQ, Guyton RA, Vinten-Johansen J, et al. Blood cardioplegia supplementation with the sodium-hydrogen ion exchange inhibitor cariporide to attenuate infarct size and coronary artery endothelial dysfunction after severe regional ischemia in a canine model. J Thorac Cardiovasc Surg. 2003;125:155-64. 